\title{
Interferência dos métodos de correção da produtividade de milho nos parâmetros de adaptabilidade e estabilidade
}

\author{
Alberto Cargnelutti Filho(1) \\ (1)Fundação Estadual de Pesquisa Agropecuária, Rua Gonçalves Dias, no 570, Bairro Menino Deus, CEP 90130-060 Porto Alegre, RS. \\ E-mail: alberto-cargnelutti@fepagro.rs.gov.br
}

\begin{abstract}
Resumo - O objetivo deste trabalho foi verificar a interferência dos métodos de correção da produtividade de grãos de milho, em função da população de plantas, nos parâmetros de adaptabilidade e estabilidade de cultivares, estimados pelo método de Lin \& Binns, modificado por Carneiro. O ajuste, ou não, da produtividade de grãos, nos 31 ensaios de competição de cultivares de milho utilizados, foi realizado com os métodos: sem correção, regra de três, método de Zuber, covariância da população média, covariância da população ideal, método de Cruz, método de Vencovsky \& Cruz e método de correção estratificada. O coeficiente de correlação de Spearman foi utilizado para verificar a magnitude da influência de cada método, em relação aos dados sem correção. Os métodos de correção proporcionam diferentes níveis de concordância nos parâmetros de adaptabilidade e estabilidade de cultivares, em relação aos dados sem correção. Os métodos de correção por análise da covariância para a população média, correção por análise de covariância para a população ideal, método proposto por Vencovsky \& Cruz e correção estratificada, são os que acarretam maiores concordâncias nos parâmetros de adaptabilidade e estabilidade de cultivares, em relação aos dados sem correção.
\end{abstract}

Termos para indexação: Zea mays, população de plantas, análise estatística, experimentação.

\section{Interference of corn yield corrections methods on adaptability and stability parameters}

\begin{abstract}
The objective of this work was to verify the interference of corn yield corrections methods, considering plants population, on the adaptability and stability parameters of corn cultivars, estimated by Lin \& Binns method modified by Carneiro. The adjustment, or not, of corn cultivars grain yield, in 31 corn trials, was made with the methods: without correction, three rule, Zuber method, covariance of the average population, covariance of the ideal population, method proposed by Cruz, method proposed by Vencovsky \& Cruz, and the method of stratified correction. The magnitude of correction influence of each method used Spearman rank correlation, in relation to the data without correction. The correction methods provide parameters to cultivars, to different levels of agreement, in the adaptability and stability in relation to data without correction. Methods of correction for covariance analysis of average and ideal populations, proposed by Vencovsky \& Cruz, and method of the stratified correction cause greater agreement on adaptability and stability parameters to cultivars, in relation to data without correction.
\end{abstract}

Index terms: Zea mays, plants population, statistic analysis, experimentation.

\section{Introdução}

A variação entre unidades experimentais, que recebem o mesmo tratamento, constitui uma estimativa de erro experimental, com interferência direta nos testes de hipótese e nos procedimentos, em relação às comparações múltiplas de médias (Ramalho et al., 2000; Storck et al., 2000a).

Em ensaios de competição de cultivares, as condições experimentais devem ser as mais uniformes possíveis. Para isso, necessita-se de cuidados na implanta- ção, na condução dos experimentos e na coleta dos dados, a fim de garantir que a superioridade de uma cultivar, em relação a outra, reflita a sua superioridade genética (Cruz \& Carneiro, 2003). O uso dos princípios básicos da experimentação no planejamento, a condução e a análise adequada de experimentos são fundamentais para se manter o erro experimental em níveis aceitáveis (Steel et al., 1997; Storck et al., 2000a; Cruz \& Carneiro, 2003).

Outras formas de se minimizar o efeito do erro experimental e discriminar melhor as diferenças, entre os 
tratamentos avaliados, são: a utilização de um maior número de repetições, juntamente com parcelas menores (Pimentel-Gomes, 1994; Conagin et al., 1995; Storck et al., 2000a); o uso de unidades experimentais homogêneas; e a realização, de maneira uniforme, dos tratos culturais inerentes à cultura, no decorrer do experimento (Lúcio, 1997). Além disso, Cruz \& Carneiro (2003) incluem a mensuração de variáveis auxiliares, ou covariáveis, relacionadas às variáveis principais, como uma das técnicas experimentais utilizadas, para reduzir a variação ambiental. Assim, a correção prévia dos dados, quanto à desuniformidade na população de plantas, consiste em uma forma de se fazer comparações mais apropriadas sobre a superioridade genotípica (Cruz \& Carneiro, 2003). A magnitude do erro experimental e o atendimento às pressuposições do modelo matemático servem para avaliar a qualidade de um experimento (Storck et al., 2000a). E ainda, as estatísticas, coeficiente de variação e diferença mínima significativa, obtida pelo teste de Tukey, são consideradas adequadas para classificar a precisão experimental (Scapim et al., 1995; Lúcio, 1997).

A precisão experimental dos ensaios de competição de cultivares de milho é baixa, ocasiona discriminação ineficiente entre as cultivares, o que pode induzir a conclusões incorretas, em relação às cultivares a serem indicadas aos produtores (Lopes, 1993; Lúcio, 1997; Marques, 1999).

A análise de covariância com a produtividade de grãos tem sido utilizada, usando-se como covariável o número de plantas na colheita (Veronesi et al., 1995; Storck et al., 2000b; Schmildt et al., 2001), os resultados de análise de solo (Feijó et al., 2001), o número de plantas e de espigas na colheita (Storck et al., 2002), buscando-se diminuir o erro experimental em ensaios de competição de cultivares de milho. Além disso, o uso de bordadura, nas extremidades das fileiras (Cargnelutti Filho et al., 2003b), e a análise espacial (Cargnelutti Filho et al., 2003a) foram estudados, a fim de reduzir o erro experimental, em ensaios de competição de cultivares de milho.

A desuniformidade, relacionada ao número de plantas por parcela, prejudica essas comparações e se constitui em um dos problemas básicos na análise e interpretação dos resultados experimentais (Schmildt et al., 2001; Cruz \& Carneiro, 2003). Por isso, tenta-se obter população uniforme pela semeadura em excesso, efetuan- do-se o desbaste para a população desejada, quando as plantas estão em estádio inicial de crescimento. Mesmo após essa prática, a parcela pode, algumas vezes, apresentar falhas por causa de fatores que fogem ao controle do pesquisador, tais como elevada precipitação pluvial, ação de pragas e doenças, entre outras, que podem afetar a realização de certas análises estatísticas, em particular, quando o caráter não é medido apenas nas plantas competitivas (Vencovsky \& Cruz, 1991). Assim, uma maneira de se considerar o efeito de população, em análises estatísticas, é incluí-lo no modelo como covariável.

Os métodos de correção da produtividade de parcelas, com populações variadas, têm sido aplicados com maior freqüência, por causa, principalmente, da disponibilidade de técnicas computacionais atuais, em relação à resolução do grande volume de cálculos que exigem. Trabalhos com dados simulados e de experimentos realizados no campo, envolvendo métodos de correção de estande, para estimar produtividade, têm sido realizados (Morais et al., 1986; Vencovsky \& Cruz, 1991; Veronesi et al., 1995; Schmildt et al., 2001; Cargnelutti Filho \& Storck, 2004). Conforme estatísticas utilizadas na verificação da eficiência dos métodos, os resultados mostram-se, em geral, promissores quanto à discriminação de cultivares, e apontam uma tendência de melhorar a precisão experimental, principalmente nos métodos que utilizam o número de plantas como covariável.

A análise de adaptabilidade e estabilidade se constitui numa das etapas finais de um programa de melhoramento de plantas, que visa indicação de cultivares para ambientes gerais e específicos de cultivo, para isso existem diversas metodologias. A princípio, o método de correção dos dados da produtividade de grãos, em experimentos inadequados quanto à população final de plantas, para ser considerado adequado, deve aumentar a discriminação de cultivares, mas não afetar a indicação, mediante análise de adaptabilidade e estabilidade. Porém, ao aplicar um método de correção, a interferência dessas correções nas estimativas dos parâmetros da análise de adaptabilidade e estabilidade, obtidos pela metodologia de Lin \& Binns (1988) modificada por Carneiro (1998), usadas para as indicações de cultivares, é pouco conhecida.

O objetivo deste trabalho foi verificar a interferência dos métodos de correção da produtividade de grãos de milho, nos parâmetros de adaptabilidade e estabilidade de cultivares. 


\section{Material e Métodos}

Foram usados dados da população final de plantas (X) e da produtividade de grãos de milho (Y), corrigidos a 13\% de umidade, de 31 ensaios de competição de cultivares de milho (Zea mays L.), realizados no Estado do Rio Grande do Sul, no ano agrícola 2002/2003.

Os ensaios foram classificados em quatro grupos de experimentos, conforme a categoria e o ciclo das cultivares, ou seja, 10 ensaios de 36 cultivares na categoria dos estaduais de ciclo precoce, nove ensaios de 11 cultivares dos estaduais do ciclo superprecoce, seis ensaios de 27 cultivares, categoria dos indicados do ciclo precoce e seis ensaios de 18 cultivares, categoria dos indicados do ciclo superprecoce. Na categoria dos ensaios estaduais, as cultivares avaliadas foram aquelas ainda não indicadas aos produtores, e as indicadas foram estudadas na categoria dos ensaios indicados.

Em todos os ensaios, as cultivares foram delineadas em blocos casualizados, com três repetições, e as unidades experimentais constituídas de duas fileiras com $5 \mathrm{~m}$ de comprimento, espaçadas em $0,8 \mathrm{~m}$. As semeaduras foram realizadas, a fim de se obter populações finais de 55.000 e 65.000 plantas ha ${ }^{-1}$, respectivamente, em relação aos ensaios dos ciclos precoce e superprecoce.

Para cada um dos 31 ensaios, foram feitas análises de variância para as variáveis $\mathrm{Y}$ e X, e a análise de covariância usando-se X como covariável. Nos casos em que o efeito da covariável $\mathrm{X}$ foi significativo, pelo teste de $F(\alpha=0,05)$, a correção dos dados da produtividade de grãos de milho foi considerada adequada.

O ajuste, ou não, da produtividade de grãos, em função da população de plantas foi feito com oito métodos, conforme Cruz \& Carneiro (2003), independentemente de ser adequado: sem correção (SC); correção por regra de três (RT); correção pelo método (Z) de Zuber (1942); correção pela covariância com a população média (CM); correção pela covariância com a população ideal (CI); correção pelo método (C) de Cruz (1971); correção pelo método (VC) de Vencovsky \& Cruz (1991); e correção estratificada (CE), proposto por Schmildt et al. (2001).

Nos ensaios em que a análise de variância, em relação à população final, apresentou significância para o efeito de cultivar, o valor da estatística "diferença mínima significativa entre as cultivares”, pelo teste de Tukey a 5\% de probabilidade, foi utilizado na obtenção dos grupos de cultivares, no método de correção estratificada.
Realizou-se, a seguir, a análise de variância conjunta da produtividade de grãos de milho, com e sem a correção dos dados, separadamente, para cada grupo de ensaios. Procedeu-se às análises de adaptabilidade e estabilidade pelo método de Lin \& Binns (1988), modificado por Carneiro (1998), usando-se os dados originais da produtividade de grãos de milho e os dados corrigidos em função da população de plantas, pelos métodos RT, Z, CM, CI, C, VC e CE. Assim, foram obtidas as estimativas dos parâmetros de estabilidade para indicação de cultivares em ambientes gerais $\left(\mathrm{P}_{\text {ig }}\right)$, grupo de ambientes favoráveis $\left(\mathrm{P}_{\text {if }}\right)$ e grupo de ambientes desfavoráveis $\left(\mathrm{P}_{\mathrm{id}}\right)$, conforme Cruz \& Carneiro (2003).

Para verificar a magnitude da influência da correção da produtividade de grãos, pelos diferentes métodos, em relação aos dados sem correção, quanto às estatísticas $\mathrm{P}_{\text {ig, }} \mathrm{P}_{\text {if }}$ e $\mathrm{P}_{\text {id }}$, foi utilizado o coeficiente de correlação classificatório de Spearman, e sua significância foi verificada por meio do teste $t$ de Student.

As análises foram feitas com o auxílio do programa GENES (Cruz, 2001) e do aplicativo Office Excel.

\section{Resultados e Discussão}

A análise de variância individual, em relação à produtividade de grãos de milho, mostrou efeito de cultivar significativo em $87 \%$ dos ensaios (27 ensaios). Aplicando-se a análise de covariância, utilizando-se a população de plantas como covariável, o percentual subiu para 94\% (29 ensaios), o que concorda com Storck et al. (2000b, 2002) e Cruz \& Carneiro (2003), que consideram essa técnica adequada para aumentar a discriminação de cultivares (Tabela 1).

Nos seis ensaios em que a estatística $\mathrm{F}(\alpha=0,05)$ da análise de variância foi significativa entre cultivares, em relação à população de plantas, revelando diferenças no comportamento das cultivares nesses ambientes, a correção dos dados de produtividade de grãos, pelo método da correção estratificada, foi considerada uma técnica adequada (Schmildt et al., 2001). Os demais 25 ensaios não apresentaram diferenças significativas entre cultivares, em relação à população de plantas, e, nesses casos, a correção da produtividade de grãos é um procedimento adequado, caso haja significância da covariável população de plantas (Steel et al., 1997; Ramalho et al., 2000; Cruz \& Carneiro, 2003). Segundo Vencovsky \& Cruz (1991) e Veronesi et al. (1995), temse realizado a correção em todos os ambientes, utilizando-se a covariância para a população ideal, por permitir 
a estabilização dos valores ajustados ao redor dessa população, que deve ser comum a todos os ensaios.

Considerando-se significante o uso da covariável população de plantas, como critério para adequabilidade da correção dos dados da produtividade de grãos, em $80 \%$, 22\%, 66\% e $100 \%$ dos ambientes, a correção foi adequada, respectivamente para ensaios dos grupos estaduais precoce, estaduais superprecoce, indicados precoce e indicados superprecoce (Tabela 1).

A análise de variância conjunta, em relação à produtividade de grãos, mostrou haver interação cultivar x ambiente, significativa nos 32 casos (quatro grupos de experimentos x oito métodos de correção), o que revela

Tabela 1. Efeito significativo $\left(\mathrm{H}_{1}\right)$ a $5 \%$ de probabilidade de erro ou não significativo $\left(\mathrm{H}_{0}\right)$, de cultivares em relação às variáveis produtividade de grãos de milho, população de plantas, produtividade de grãos corrigido (PGC) e à covariável população de plantas (Covar-Pop), e situação recomendada nos quatro grupos de experimentos.

\begin{tabular}{|c|c|c|c|c|c|}
\hline \multirow[t]{2}{*}{ Ensaio } & \multicolumn{2}{|c|}{ Análise de variância } & \multicolumn{2}{|c|}{ Análise de covariância } & \multirow[t]{2}{*}{ Situação ${ }^{(1)}$} \\
\hline & Produtividade & População & PGC & Covar-Pop & \\
\hline & \multicolumn{5}{|c|}{ Estadual precoce } \\
\hline 1 & $\mathrm{H}_{1}$ & $\mathrm{H}_{1}$ & $\mathrm{H}_{1}$ & $\mathrm{H}_{1}$ & Sim \\
\hline 2 & $\mathrm{H}_{1}$ & $\mathrm{H}_{0}$ & $\mathrm{H}_{1}$ & $\mathrm{H}_{1}$ & Sim \\
\hline 3 & $\mathrm{H}_{1}$ & $\mathrm{H}_{1}$ & $\mathrm{H}_{1}$ & $\mathrm{H}_{1}$ & Sim \\
\hline 4 & $\mathrm{H}_{1}$ & $\mathrm{H}_{0}$ & $\mathrm{H}_{1}$ & $\mathrm{H}_{1}$ & Sim \\
\hline 5 & $\mathrm{H}_{1}$ & $\mathrm{H}_{0}$ & $\mathrm{H}_{1}$ & $\mathrm{H}_{1}$ & Sim \\
\hline 6 & $\mathrm{H}_{1}$ & $\mathrm{H}_{1}$ & $\mathrm{H}_{1}$ & $\mathrm{H}_{1}$ & Sim \\
\hline 7 & $\mathrm{H}_{1}$ & $\mathrm{H}_{0}$ & $\mathrm{H}_{1}$ & $\mathrm{H}_{0}$ & Não \\
\hline 8 & $\mathrm{H}_{1}$ & $\mathrm{H}_{0}$ & $\mathrm{H}_{1}$ & $\mathrm{H}_{1}$ & Sim \\
\hline 9 & $\mathrm{H}_{1}$ & $\mathrm{H}_{0}$ & $\mathrm{H}_{1}$ & $\mathrm{H}_{0}$ & Não \\
\hline 10 & $\mathrm{H}_{1}$ & $\mathrm{H}_{0}$ & $\mathrm{H}_{1}$ & $\mathrm{H}_{1}$ & Sim \\
\hline \multicolumn{6}{|c|}{ Estadual superprecoce } \\
\hline 1 & $\mathrm{H}_{1}$ & $\mathrm{H}_{0}$ & $\mathrm{H}_{1}$ & $\mathrm{H}_{0}$ & Não \\
\hline 2 & $\mathrm{H}_{1}$ & $\mathrm{H}_{0}$ & $\mathrm{H}_{1}$ & $\mathrm{H}_{0}$ & Não \\
\hline 3 & $\mathrm{H}_{1}$ & $\mathrm{H}_{0}$ & $\mathrm{H}_{1}$ & $\mathrm{H}_{0}$ & Não \\
\hline 4 & $\mathrm{H}_{1}$ & $\mathrm{H}_{0}$ & $\mathrm{H}_{1}$ & $\mathrm{H}_{0}$ & Não \\
\hline 5 & $\mathrm{H}_{1}$ & $\mathrm{H}_{1}$ & $\mathrm{H}_{1}$ & $\mathrm{H}_{1}$ & Sim \\
\hline 6 & $\mathrm{H}_{0}$ & $\mathrm{H}_{1}$ & $\mathrm{H}_{1}$ & $\mathrm{H}_{1}$ & Sim \\
\hline 7 & $\mathrm{H}_{1}$ & $\mathrm{H}_{0}$ & $\mathrm{H}_{1}$ & $\mathrm{H}_{0}$ & Não \\
\hline 8 & $\mathrm{H}_{1}$ & $\mathrm{H}_{0}$ & $\mathrm{H}_{1}$ & $\mathrm{H}_{0}$ & Não \\
\hline 9 & $\mathrm{H}_{1}$ & $\mathrm{H}_{0}$ & $\mathrm{H}_{1}$ & $\mathrm{H}_{0}$ & Não \\
\hline \multicolumn{6}{|c|}{ Indicado precoce } \\
\hline 1 & $\mathrm{H}_{1}$ & $\mathrm{H}_{0}$ & $\mathrm{H}_{1}$ & $\mathrm{H}_{1}$ & Sim \\
\hline 2 & $\mathrm{H}_{1}$ & $\mathrm{H}_{0}$ & $\mathrm{H}_{1}$ & $\mathrm{H}_{1}$ & Sim \\
\hline 3 & $\mathrm{H}_{1}$ & $\mathrm{H}_{0}$ & $\mathrm{H}_{1}$ & $\mathrm{H}_{1}$ & Sim \\
\hline 4 & $\mathrm{H}_{1}$ & $\mathrm{H}_{0}$ & $\mathrm{H}_{1}$ & $\mathrm{H}_{0}$ & Não \\
\hline 5 & $\mathrm{H}_{1}$ & $\mathrm{H}_{0}$ & $\mathrm{H}_{1}$ & $\mathrm{H}_{0}$ & Não \\
\hline 6 & $\mathrm{H}_{1}$ & $\mathrm{H}_{0}$ & $\mathrm{H}_{1}$ & $\mathrm{H}_{1}$ & Sim \\
\hline \multicolumn{6}{|c|}{ Indicado superprecoce } \\
\hline 1 & $\mathrm{H}_{1}$ & $\mathrm{H}_{0}$ & $\mathrm{H}_{1}$ & $\mathrm{H}_{1}$ & Sim \\
\hline 2 & $\mathrm{H}_{0}$ & $\mathrm{H}_{0}$ & $\mathrm{H}_{1}$ & $\mathrm{H}_{1}$ & Sim \\
\hline 3 & $\mathrm{H}_{1}$ & $\mathrm{H}_{1}$ & $\mathrm{H}_{1}$ & $\mathrm{H}_{1}$ & Sim \\
\hline 4 & $\mathrm{H}_{0}$ & $\mathrm{H}_{0}$ & $\mathrm{H}_{0}$ & $\mathrm{H}_{1}$ & Sim \\
\hline 5 & $\mathrm{H}_{0}$ & $\mathrm{H}_{0}$ & $\mathrm{H}_{0}$ & $\mathrm{H}_{1}$ & Sim \\
\hline 6 & $\mathrm{H}_{1}$ & $\mathrm{H}_{0}$ & $\mathrm{H}_{1}$ & $\mathrm{H}_{1}$ & Sim \\
\hline
\end{tabular}

(1)Sim: proceder à correção da produtividade de grãos, quando o efeito da covariável população de plantas for significativo $\left(\mathrm{H}_{1}\right)$; Não: em caso contrário $\left(\mathrm{H}_{0}\right)$. comportamento diferenciado das cultivares nos ambientes, e que a indicação de cultivares, por meio da análise de adaptabilidade e estabilidade, é um procedimento adequado (Tabela 2). De maneira geral, conforme Pimentel-Gomes (1990), os ensaios estaduais precoce e indicados precoce apresentaram variâncias residuais homogêneas, com exceção dos dados de produtividade de grãos, obtidos com os métodos de correção por regra de três, método de Zuber e método de Cruz nos indicados precoce, enquanto os estaduais superprecoce e indicados superprecoce revelaram variâncias residuais heterogêneas, indicando necessidade de exclusão de ensaio(os) com quadrado médio residual discrepante, formação de grupos de ensaios com variâncias residuais homogêneas ou ajuste dos graus de liberdade, para interpretação correta dos testes de hipóteses (Tabela 3).

A relação entre o maior e o menor quadrado médio residual da produtividade de grãos, após a exclusão dos ensaios 5 e 6, no grupo dos estaduais superprecoces, e o ensaio 3 nos indicados superprecoces, oscilou entre 4,64 a 4,75 nos estaduais superprecoces, e 4,25 a 5,41 nos indicados superprecoces, o que revelou variâncias residuais homogêneas conforme critérios de Pimentel-Gomes (1990). No entanto, para este estudo foram considerados todos os ensaios, com o objetivo de se discutir as interferências da heterogeneidade das variâncias residuais, nos parâmetros de adaptabilidade e estabilidade.

Usando-se o índice ambiental (diferença entre a média de produtividade de grãos das cultivares em cada local e a média geral), como critério para classificação dos ambientes em favorável (índice ambiental positivo) e desfavorável (índice ambiental negativo), os métodos de correção não alteraram a classificação dos ambientes, em relação aos dados sem correção, no grupo de experimentos com homogeneidade das variâncias residuais (estaduais precoce e indicados precoce), com exceção do método de correção por regra de três, que enquadrou o ambiente 6 , do grupo dos estaduais precoce, como favorável (índice ambiental $=0,507 \mathrm{t} \mathrm{ha}^{-1}$ ), enquanto que na análise com os dados originais tal ambiente foi classificado como desfavorável (índice ambiental $=-1,639 \mathrm{t} \mathrm{ha}^{-1}$ ) $($ Tabela 4).

Essa alteração pode ser explicada, provavelmente, pela inexistência de proporcionalidade linear entre o número de plantas e a produtividade de grãos observada, em que esta última pode ser superestimada por esse método de correção (Vencovsky \& Cruz, 1991; Veronesi et al., 1995). 
Nos grupos de experimentos com variâncias residuais heterogêneas (estaduais superprecoce e indicados superprecoce), ocorreram maiores mudanças na classificação dos ambientes. Ao comparar os parâmetros de

Tabela 2. Análise de variância conjunta da produtividade de grãos, nos quatro grupos de experimentos de cultivares de milho e nos diferentes métodos de correção.

\begin{tabular}{|c|c|c|c|c|c|c|c|c|c|}
\hline \multirow[t]{2}{*}{ Causa de variação } & \multirow[t]{2}{*}{ GL } & \multicolumn{8}{|c|}{ Método de correção ${ }^{(1)}$} \\
\hline & & $\mathrm{SC}$ & RT & Z & $\mathrm{CM}$ & $\mathrm{CI}$ & $\mathrm{C}$ & $\mathrm{VC}$ & $\mathrm{CE}$ \\
\hline & & \multicolumn{8}{|c|}{ Estadual precoce } \\
\hline Blocos/Amb & 20 & 2,196 & 2,865 & 2,485 & 2,243 & 2,243 & 2,274 & 2,276 & 2,078 \\
\hline Blocos & 2 & 0,189 & 0,534 & 0,146 & 0,135 & 0,135 & 0,044 & 0,061 & 0,154 \\
\hline $\mathrm{B} 1 \times \mathrm{Amb}$ & 18 & 2,419 & 3,124 & 2,745 & 2,477 & 2,477 & 2,522 & 2,522 & 2,292 \\
\hline Cultivar & 35 & $34,066^{*}$ & $30,424 *$ & $31,267^{*}$ & $30,172 *$ & $30,172 *$ & $32,334 *$ & $32,178^{*}$ & $30,342 *$ \\
\hline Ambientes & 9 & $495,353 *$ & $363,515^{*}$ & $380,926^{*}$ & $495,353^{*}$ & $406,241 *$ & $399,666^{*}$ & $407,383^{*}$ & $406,323^{*}$ \\
\hline Cult x Amb & 315 & $2,492 *$ & $2,616^{*}$ & $2,437 *$ & $2,383 *$ & $2,383^{*}$ & $2,617 *$ & $2,539 *$ & $2,393^{*}$ \\
\hline Resíduo & 700 & 0,844 & 0,847 & 0,751 & 0,678 & 0,678 & 0,744 & 0,733 & 0,689 \\
\hline Média & - & 7,405 & 7,637 & 7,568 & 7,405 & 7,629 & 7,603 & 7,635 & 7,630 \\
\hline \multirow[t]{2}{*}{$\mathrm{CV}(\%)$} & - & 12,40 & 12,05 & 11,45 & 11,12 & 10,80 & 11,34 & 11,21 & 10,87 \\
\hline & & \multicolumn{8}{|c|}{ Estadual superprecoce } \\
\hline Blocos/Amb & 18 & 1,974 & 2,478 & 2,228 & 1,982 & 1,982 & 2,372 & 1,983 & 1,885 \\
\hline Blocos & 2 & 1,512 & 0,484 & 0,706 & 0,549 & 0,549 & 0,354 & 0,569 & 0,813 \\
\hline $\mathrm{B} 1 \times \mathrm{Amb}$ & 16 & 2,031 & 2,727 & 2,418 & 2,161 & 2,161 & 2,624 & 2,159 & 2,019 \\
\hline Cultivar & 10 & $34,787 *$ & $25,438^{*}$ & $27,471^{*}$ & $26,436^{*}$ & $26,436^{*}$ & $28,914 *$ & $27,235^{*}$ & $30,228^{*}$ \\
\hline Ambientes & 8 & $130,979 *$ & $106,401 *$ & $109,696^{*}$ & $130,979 *$ & $119,710^{*}$ & $130,845^{*}$ & $119,566^{*}$ & $119,419^{*}$ \\
\hline Cult x Amb & 80 & $1,806^{*}$ & $1,858^{*}$ & $1,721 *$ & $2,042 *$ & $2,042 *$ & $2,414 *$ & $2,074 *$ & $2,130^{*}$ \\
\hline Resíduo & 180 & 0,769 & 0,955 & 0,800 & 0,554 & 0,554 & 0,709 & 0,677 & 0,644 \\
\hline Média & - & 7,519 & 8,235 & 8,020 & 7,519 & 7,875 & 7,810 & 7,869 & 7,797 \\
\hline \multirow[t]{2}{*}{$\mathrm{CV}(\%)$} & - & 11,66 & 11,86 & 11,15 & 9,90 & 9,45 & 10,78 & 10,45 & 10,29 \\
\hline & & \multicolumn{8}{|c|}{ Indicado precoce } \\
\hline Blocos/Amb & 12 & 3,175 & 3,093 & 2,993 & 2,915 & 2,915 & 3,472 & 2,841 & 2,915 \\
\hline Blocos & 2 & 1,854 & 3,431 & 2,837 & 1,767 & 1,767 & 3,349 & 1,982 & 1,767 \\
\hline $\mathrm{Bl} \times \mathrm{Amb}$ & 10 & 3,439 & 3,026 & 3,024 & 3,144 & 3,144 & 3,497 & 3,013 & 3,144 \\
\hline Cultivar & 26 & $14,054^{*}$ & $12,591^{*}$ & $12,750^{*}$ & $11,868^{*}$ & $11,868^{*}$ & $13,942 *$ & $12,386^{*}$ & $11,868^{*}$ \\
\hline Ambientes & 5 & $287,315^{*}$ & $105,904 *$ & $144,436^{*}$ & $287,315^{*}$ & $170,728 *$ & $178,309 *$ & $169,201 *$ & $170,728^{*}$ \\
\hline Cult x Amb & 130 & $2,651 *$ & $3,491 *$ & $2,972 *$ & $2,419^{*}$ & $2,419^{*}$ & $2,762 *$ & $2,536^{*}$ & $2,419^{*}$ \\
\hline Resíduo & 312 & 1,074 & 1,680 & 1,261 & 0,901 & 0,901 & 1,224 & 0,960 & 0,901 \\
\hline Média & - & 7,801 & 7,832 & 7,823 & 7,801 & 7,757 & 7,683 & 7,770 & 7,757 \\
\hline $\mathrm{CV}(\%)$ & - & 13,29 & 16,55 & 14,35 & 12,17 & 12,23 & 14,40 & 12,61 & 12,23 \\
\hline \multicolumn{10}{|c|}{ Indicado superprecoce } \\
\hline Blocos/Amb & 12 & 1,834 & 2,603 & 2,164 & 1,590 & 1,590 & 2,387 & 2,111 & 1,560 \\
\hline Blocos & 2 & 0,330 & 1,226 & 0,859 & 0,773 & 0,773 & 1,702 & 1,372 & 0,826 \\
\hline $\mathrm{Bl} \times \mathrm{Amb}$ & 10 & 2,135 & 2,878 & 2,425 & 1,754 & 1,754 & 2,524 & 2,259 & 1,707 \\
\hline Cultivar & 17 & $10,131^{*}$ & $6,769^{*}$ & $7,446^{*}$ & $5,721 *$ & $5,721^{*}$ & $7,226^{*}$ & $6,949 *$ & $5,953^{*}$ \\
\hline Ambientes & 5 & $292,628^{*}$ & $163,540 *$ & $196,594^{*}$ & $292,628^{*}$ & $180,426^{*}$ & $186,664^{*}$ & $183,681^{*}$ & $179,704^{*}$ \\
\hline Cult x Amb & 85 & $2,601 *$ & $2,653^{*}$ & $2,549 *$ & $2,195^{*}$ & $2,195^{*}$ & $2,755^{*}$ & $2,607 *$ & $2,255^{*}$ \\
\hline Resíduo & 204 & 1,881 & 1,726 & 1,660 & 1,450 & 1,450 & 1,692 & 1,660 & 1,452 \\
\hline Média & - & 7,940 & 8,624 & 8,419 & 7,940 & 8,478 & 8,465 & 8,464 & 8,468 \\
\hline $\mathrm{CV}(\%)$ & - & 17,27 & 15,23 & 15,30 & 15,17 & 14,20 & 15,37 & 15,22 & 14,23 \\
\hline
\end{tabular}

(1)SC: sem correção; RT: correção por regra de três; Z: correção pelo método de Zuber (1942); CM: correção pela covariância com população média; CI: correção pela covariância com população ideal; C: correção pelo método de Cruz (1971); VC: correção pelo método de Vencovsky \& Cruz (1991); CE: correção estratificada. *Significativo a 5\% de probabilidade pelo teste F.

Tabela 3. Valores da relação entre o maior e o menor quadrado médio residual, quanto à análise da produtividade de grãos de milho, nos quatro grupos de experimentos de cultivares de milho e nos diferentes métodos de correção.

\begin{tabular}{|c|c|c|c|c|c|c|c|c|}
\hline \multirow[t]{2}{*}{ Grupo de experimento } & \multicolumn{8}{|c|}{ Método de correção ${ }^{(1)}$} \\
\hline & $\mathrm{SC}$ & RT & $\mathrm{Z}$ & $\mathrm{CM}$ & CI & $\mathrm{C}$ & $\mathrm{VC}$ & $\mathrm{CE}$ \\
\hline Estadual precoce & 4,51 & 4,12 & 4,78 & 4,94 & 4,94 & 4,88 & 4,84 & 4,94 \\
\hline Estadual superprecoce & 15,92 & 8,51 & 10,79 & 7,99 & 7,99 & 9,14 & 11,44 & 14,13 \\
\hline Indicado precoce & 2,87 & 13,60 & 9,93 & 4,58 & 4,58 & 9,34 & 5,61 & 4,58 \\
\hline Indicado superprecoce & 20,06 & 14,55 & 17,19 & 17,19 & 17,19 & 18,44 & 19,09 & 16,39 \\
\hline
\end{tabular}

(1)SC: sem correção; RT: correção por regra de três; Z: correção pelo método de Zuber (1942); CM: correção pela covariância com população média; CI: correção pela covariância com população ideal; C: correção pelo método de Cruz (1971); VC: correção pelo método de Vencovsky \& Cruz (1991); CE: correção estratificada. 
desempenho em ambiente geral $\left(\mathrm{P}_{\mathrm{ig}}\right)$, em ambientes favoráveis $\left(\mathrm{P}_{\mathrm{if}}\right)$ e em desfavoráveis $\left(\mathrm{P}_{\mathrm{id}}\right)$, obtidos com a análise de estabilidade pelo método de Lin \& Binns (1988), modificado por Carneiro (1998), nos sete métodos de correção com os dados sem ajuste, essas alterações na classificação dos ambientes poderão interferir nos níveis de concordância da indicação de cultivares.

Coeficientes de correlação de Spearman igual a 1 e -1 indicam, respectivamente, total concordância e total discordância dos postos classificatórios entre duas variáveis. Ao comparar os postos classificatórios das cultivares nos ambientes gerais $\left(\mathrm{P}_{\mathrm{ig}}\right)$, favoráveis $\left(\mathrm{P}_{\mathrm{if}}\right)$ e desfavoráveis $\left(\mathrm{P}_{\mathrm{id}}\right)$, obtidos nos sete métodos de correção da produtividade de grãos, com os dados sem o ajuste prévio, nos quatro grupos de experimentos, o coeficiente de correlação de Spearman variou de 0,5636 a 1,0000, o que mostra níveis distintos de concordância na classi-

Tabela 4. Valores dos índices ambientais $\left(t \mathrm{tha}^{-1}\right)$, em relação à análise conjunta da produtividade de grãos de milho $\left(\mathrm{t} \mathrm{ha}^{-1}\right)$, sem e com correção da população de plantas, nos quatro grupos de experimentos.

\begin{tabular}{|c|c|c|c|c|c|c|c|c|}
\hline \multirow[t]{2}{*}{ Ensaio } & \multicolumn{8}{|c|}{ Método de correção ${ }^{(1)}$} \\
\hline & $\mathrm{SC}$ & RT & $\mathrm{Z}$ & $\mathrm{CM}$ & $\mathrm{CI}$ & $\mathrm{C}$ & $\mathrm{VC}$ & $\mathrm{CE}$ \\
\hline & \multicolumn{8}{|c|}{ Estadual precoce } \\
\hline 1 & 0,334 & 0,246 & 0,272 & 0,334 & 0,426 & 0,453 & 0,395 & 0,396 \\
\hline 2 & $-1,603$ & $-1,410$ & $-1,468$ & $-1,603$ & $-1,606$ & $-1,582$ & $-1,604$ & $-1,608$ \\
\hline 3 & $-2,179$ & $-2,195$ & $-2,191$ & $-2,179$ & $-2,170$ & $-2,153$ & $-2,182$ & $-2,181$ \\
\hline 4 & 3,563 & 1,555 & 2,157 & 3,563 & 2,548 & 2,495 & 2,559 & 2,547 \\
\hline 5 & 1,469 & 1,438 & 1,447 & 1,469 & 1,372 & 1,384 & 1,376 & 1,371 \\
\hline 6 & $-1,639$ & 0,507 & $-0,137$ & $-1,639$ & $-0,150$ & $-0,275$ & $-0,108$ & $-0,099$ \\
\hline 7 & $-0,371$ & $-0,510$ & $-0,468$ & $-0,371$ & $-0,580$ & $-0,556$ & $-0,586$ & $-0,581$ \\
\hline 8 & 1,505 & 1,530 & 1,523 & 1,505 & 1,363 & 1,377 & 1,360 & 1,362 \\
\hline 9 & $-3,121$ & $-3,356$ & $-3,285$ & $-3,121$ & $-3,347$ & $-3,300$ & $-3,351$ & $-3,349$ \\
\hline \multirow[t]{2}{*}{10} & 2,041 & 2,196 & 2,149 & 2,041 & 2,142 & 2,157 & 2,141 & 2,141 \\
\hline & \multicolumn{8}{|c|}{ Estadual superprecoce } \\
\hline 1 & 1,169 & 1,058 & 1,091 & 1,169 & 0,898 & 0,941 & 0,907 & 0,976 \\
\hline 2 & $-2,749$ & $-1,477$ & $-1,859$ & $-2,749$ & $-2,540$ & $-3,023$ & $-2,526$ & $-2,462$ \\
\hline 3 & 2,874 & 2,093 & 2,327 & 2,874 & 2,504 & 2,583 & 2,512 & 2,582 \\
\hline 4 & 0,151 & $-0,255$ & $-0,133$ & 0,151 & $-0,165$ & $-0,121$ & $-0,159$ & $-0,087$ \\
\hline 5 & $-0,526$ & 0,799 & 0,401 & $-0,526$ & 0,564 & 0,461 & 0,584 & 0,457 \\
\hline 6 & $-0,452$ & $-0,755$ & $-0,664$ & $-0,452$ & 0,103 & 0,286 & 0,025 & $-0,334$ \\
\hline 7 & 1,744 & 1,406 & 1,508 & 1,744 & 1,499 & 1,559 & 1,506 & 1,577 \\
\hline 8 & $-3,176$ & $-3,753$ & $-3,580$ & $-3,176$ & $-3,528$ & $-3,426$ & $-3,520$ & $-3,450$ \\
\hline 9 & 0,965 & 0,884 & 0,908 & 0,965 & 0,664 & 0,740 & 0,671 & 0,742 \\
\hline \multicolumn{9}{|c|}{ Indicado precoce } \\
\hline 1 & $-1,534$ & $-1,121$ & $-1,245$ & $-1,534$ & $-1,192$ & $-1,141$ & $-1,209$ & $-1,192$ \\
\hline 2 & 3,163 & 1,202 & 1,790 & 3,163 & 1,939 & 1,926 & 1,933 & 1,939 \\
\hline 3 & 0,906 & 1,147 & 1,075 & 0,906 & 1,150 & 1,207 & 1,151 & 1,150 \\
\hline 4 & $-1,118$ & $-0,596$ & $-0,753$ & $-1,118$ & $-0,958$ & $-1,105$ & $-0,917$ & $-0,958$ \\
\hline 5 & $-1,781$ & $-1,308$ & $-1,450$ & $-1,781$ & $-1,626$ & $-1,652$ & $-1,624$ & $-1,626$ \\
\hline 6 & 0,365 & 0,676 & 0,582 & 0,365 & 0,687 & 0,765 & 0,666 & 0,687 \\
\hline \multicolumn{9}{|c|}{ Indicado superprecoce } \\
\hline 1 & $-3,518$ & $-2,262$ & $-2,639$ & $-3,518$ & $-2,664$ & $-2,728$ & $-2,662$ & $-2,653$ \\
\hline 2 & 2,623 & 1,727 & 1,996 & 2,623 & 1,885 & 1,907 & 1,906 & 1,896 \\
\hline 3 & 0,917 & 0,656 & 0,734 & 0,917 & 0,552 & 0,558 & 0,563 & 0,499 \\
\hline 4 & $-1,812$ & $-1,880$ & $-1,859$ & $-1,812$ & $-1,712$ & $-1,751$ & $-1,755$ & $-1,701$ \\
\hline 5 & $-0,133$ & 0,003 & $-0,038$ & $-0,133$ & 0,282 & 0,373 & 0,268 & 0,293 \\
\hline 6 & 1,923 & 1,756 & 1,806 & 1,923 & 1,656 & 1,641 & 1,680 & 1,666 \\
\hline
\end{tabular}

(1)SC: sem correção; RT: correção por regra de três; Z: correção pelo método de Zuber (1942); CM: correção pela covariância com população média; CI: correção pela covariância com população ideal; C: correção pelo método de Cruz (1971); VC: correção pelo método de Vencovsky \& Cruz (1991); CE: correção estratificada. ficação das cultivares. Com exceção dos coeficientes 0,5636 e 0,6818 , no grupo dos ensaios estaduais superprecoce, em relação aos ambientes desfavoráveis, que foram, respectivamente, não significativo e significativo a $5 \%$ de probabilidade de erro, pelo teste t de Student, os demais valores foram significativos $(\alpha=0,01)$ (Tabela 5).

Tabela 5. Estimativas do coeficiente de correlação classificatório de Spearman, em relação aos parâmetros de estabilidade do método de Lin \& Binns (1988), modificado por Carneiro (1998), entre os dados sem correção da população de plantas e os dados ajustados por diferentes métodos de correção, nos quatro grupos de experimentos de cultivares de milho.

\begin{tabular}{|c|c|c|c|}
\hline Método $^{(1)}$ & $\begin{array}{c}\text { Geral } \\
\left(\mathrm{P}_{\mathrm{ig}}\right) \\
\end{array}$ & $\begin{array}{c}\text { Ambientes favoráveis } \\
\left(\mathrm{P}_{\mathrm{if}}\right)\end{array}$ & $\begin{array}{c}\text { Ambientes desfavoráveis } \\
\left(\mathrm{P}_{\mathrm{id}}\right)\end{array}$ \\
\hline & & Estadual precoce & \\
\hline RT & $0,9748 * *$ & $0,9560^{* *}$ & $0,9611^{* *}$ \\
\hline Z & $0,9882 * *$ & $0,9900^{* *}$ & $0,9529 * *$ \\
\hline $\mathrm{CM}$ & $0,9933 * *$ & $0,9943 * *$ & $0,9619^{* *}$ \\
\hline CI & $0,9933 * *$ & $0,9943 * *$ & $0,9619^{* *}$ \\
\hline $\mathrm{C}$ & $0,9864 * *$ & $0,9912 * *$ & $0,9349 * *$ \\
\hline $\mathrm{VC}$ & $0,9876^{* *}$ & $0,9936^{* *}$ & $0,9447^{* *}$ \\
\hline $\mathrm{CE}$ & $0,9918 * *$ & $0,9943 * *$ & $0,9717^{* *}$ \\
\hline \multirow[t]{2}{*}{ Média } & 0,9879 & 0,9877 & 0,9556 \\
\hline & & Estadual superprecoce & \\
\hline RT & $0,8000^{* *}$ & $0,8667 * *$ & $0,5636^{\mathrm{ns}}$ \\
\hline Z & $0,9364 * *$ & $0,9636^{* *}$ & $0,6818^{*}$ \\
\hline $\mathrm{CM}$ & $0,9364 * *$ & $1,0000 * *$ & $0,6818^{*}$ \\
\hline CI & $0,9364 * *$ & $0,9515^{* *}$ & $0,7455^{* *}$ \\
\hline $\mathrm{C}$ & $0,9455^{* *}$ & $0,8788^{* *}$ & $0,7818^{* *}$ \\
\hline $\mathrm{VC}$ & $0,9455^{* *}$ & $0,9636 * *$ & $0,7455^{* *}$ \\
\hline $\mathrm{CE}$ & $1,0000^{* *}$ & $0,9636^{* *}$ & $0,7636^{* *}$ \\
\hline \multirow[t]{2}{*}{ Média } & 0,9286 & 0,9411 & 0,7091 \\
\hline & & Indicado precoce & \\
\hline RT & $0,9487 * *$ & $0,9713 * *$ & $0,8828^{* *}$ \\
\hline Z & $0,9725^{* *}$ & $0,9823 * *$ & $0,9426^{* *}$ \\
\hline $\mathrm{CM}$ & $0,9853 * *$ & $0,9896 * *$ & $0,9811^{* *}$ \\
\hline CI & $0,9853 * *$ & $0,9896^{* *}$ & $0,9811^{* *}$ \\
\hline $\mathrm{C}$ & $0,9884 * *$ & $0,9817 * *$ & $0,9432 * *$ \\
\hline $\mathrm{VC}$ & $0,9841 * *$ & $0,9853 * *$ & $0,9805^{* *}$ \\
\hline $\mathrm{CE}$ & $0,9853 * *$ & $0,9896^{* *}$ & $0,9811^{* *}$ \\
\hline \multirow[t]{2}{*}{ Média } & 0,9785 & 0,9842 & 0,9560 \\
\hline & & Indicado superprecoce & \\
\hline RT & $0,8514 * *$ & $0,8989 * *$ & $0,8431^{* *}$ \\
\hline $\mathrm{Z}$ & $0,9112 * *$ & $0,9794 * *$ & $0,8596^{* *}$ \\
\hline $\mathrm{CM}$ & $0,9732 * *$ & $0,9856 * *$ & $0,8452 * *$ \\
\hline CI & $0,9732 * *$ & $0,9670 * *$ & $0,8576^{* *}$ \\
\hline $\mathrm{C}$ & $0,8906 * *$ & $0,9670^{* *}$ & $0,8658^{* *}$ \\
\hline $\mathrm{VC}$ & $0,9112 * *$ & $0,9628 * *$ & $0,8679 * *$ \\
\hline $\mathrm{CE}$ & $0,9732 * *$ & $0,9670 * *$ & $0,8576^{* *}$ \\
\hline \multirow[t]{2}{*}{ Média } & 0,9263 & 0,9611 & 0,8567 \\
\hline & \multicolumn{3}{|c|}{ Média dos quatro grupos de experimentos } \\
\hline RT & 0,8937 & 0,9232 & 0,8127 \\
\hline Z & 0,9521 & 0,9788 & 0,8592 \\
\hline $\mathrm{CM}$ & 0,9720 & 0,9924 & 0,8675 \\
\hline $\mathrm{CI}$ & 0,9720 & 0,9756 & 0,8865 \\
\hline C & 0,9527 & 0,9547 & 0,8814 \\
\hline $\mathrm{VC}$ & 0,9571 & 0,9763 & 0,8846 \\
\hline $\mathrm{CE}$ & 0,9876 & 0,9786 & 0,8935 \\
\hline Média & 0,9553 & 0,9685 & 0,8694 \\
\hline
\end{tabular}

(1)RT: correção por regra de três; Z: correção pelo método de Zuber (1942); CM: correção pela covariância com população média; CI: correção pela covariância com população ideal; C: correção pelo método de Cruz (1971); VC: correção pelo método de Vencovsky \& Cruz (1991); CE: correção estratificada. nsNão-significativo. * e **Significativo a $5 \%$ e $1 \%$, pelo teste t, com 34, 9, 25 e 16 graus de liberdade, respectivamente, para os ensaios estaduais precoce, estaduais superprecoce, indicados precoce e indicados superprecoce. 
Nos quatro grupos de experimentos, ao comparar cada método de correção em relação aos dados sem correção, houve maior concordância na indicação de cultivares (maior coeficiente de correlação de Spearman), quanto ao desempenho geral e em ambientes favoráveis. A indicação para esses ambientes parece não sofrer interferência da adequabilidade da correção dos dados, da heterogeneidade das variâncias residuais e das alterações na classificação dos ambientes. Menor concordância ocorreu na indicação de cultivares para os ambientes desfavoráveis. Nesses, menores valores do coeficiente de correlação de Spearman coincidiram com os grupos de experimentos com variâncias residuais heterogêneas, e com as maiores alterações nos índices ambientais (estaduais superprecoce e indicados superprecoce).

Entre esses dois grupos de experimentos, a maior redução do coeficiente de correlação de Spearman, no estadual superprecoce, pode ser explicada pela maior porcentagem (78\%) dos ensaios, com ajuste dos dados inadequados, pelas variâncias residuais heterogêneas, e pelo maior nível de alterações nas classificações dos ambientes, quando comparados ao grupo indicado superprecoce.

No grupo de experimentos com adequabilidade da correção dos dados, com variâncias residuais homogêneas, e sem alterações nos índices ambientais (estaduais precoce e indicados precoce), ocorreu maior concordância na indicação de cultivares em relação aos dados originais para os ambientes desfavoráveis.

Quando a correção foi adequada e as variâncias residuais foram homogêneas, os métodos de correção acarretaram menores alterações na indicação de cultivares, quanto ao desempenho geral e em ambiente favorável e desfavorável.

Quando houve inadequabilidade da correção dos dados, associada a variâncias residuais heterogêneas, ocorreu menor concordância na indicação de cultivares para os ambientes desfavoráveis.

De maneira geral, os métodos de correção por regra de três (RT), método de Zuber (Z) e o método proposto por Cruz (C) proporcionaram a menor concordância (menores valores do coeficiente de correlação de Spearman) dos parâmetros $P_{\text {ig }}, P_{\text {if }}$ e $P_{\text {id, }}$, em relação aos dados originais, quando comparados aos métodos de correção por análise de covariância para a população média (CM), correção por análise de covariância para a população ideal (CI), correção pelo método de Vencovsky \& Cruz (VC) e correção estratificada (CE), nos quatro grupos de experimentos (Tabela 5).
Cargnelutti Filho \& Storck (2004), utilizando os mesmos dados deste trabalho, verificaram que os métodos CM, CI, VC e CE foram eficientes em melhorar a discriminação de cultivares de milho, em relação aos dados sem correção, enquanto os métodos de RT, Z e C foram considerados pouco adequados à correção dos dados, o que concorda com Schmildt et al. (2001). Os métodos CM (Morais et al., 1986), CI (Morais et al., 1986; Vencovsky \& Cruz, 1991; Veronesi et al., 1995) e VC (Vencovsky \& Cruz, 1991; Veronesi et al., 1995) são apontados como os mais eficientes em termos de correção da produtividade de grãos, enquanto os métodos RT (Vencovsky \& Cruz, 1991; Veronesi et al., 1995) e Z (Morais et al., 1986; Veronesi et al., 1995) são considerados inadequados.

Os métodos que envolvem análise de covariância para a população média (CM), covariância para a população ideal (CI), e o que se baseia em um fator de compensação estimado a partir dos dados experimentais (VC), além da correção estratificada (CE), proporcionam maiores discriminações de cultivares, e acarretam menor discordância na indicação de cultivares por meio dos parâmetros de estabilidade $\left(\mathrm{P}_{\text {igg }}, \mathrm{P}_{\text {if }}\right.$ e $\left.\mathrm{P}_{\text {id }}\right)$, da metodologia de Lin \& Binns (1988), modificada por Carneiro (1998), em relação aos dados sem correção; do ponto de vista de técnicas experimentais, esses devem ser preferidos, quando a correção prévia dos dados da produtividade de grãos, em função da população de plantas, for realizada.

\section{Conclusões}

1. Os métodos de correção da produtividade de grãos, que utilizam os dados da população final de plantas, proporcionam distintos níveis de concordância, nos parâmetros de adaptabilidade e estabilidade de cultivares de milho, em relação aos dados sem correção.

2. Os métodos de correção por análise de covariância para a população média, correção por análise de covariância para a população ideal, método de Vencovsky \& Cruz e correção estratificada são os que acarretam maior concordância com os parâmetros de adaptabilidade e estabilidade de cultivares, do método de Lin \& Binns modificado por Carneiro, em relação aos dados sem correção, e devem ser preferidos, quando a correção prévia dos dados da produtividade de grãos, em função da população de plantas, for realizada. 


\section{Agradecimentos}

Ao Engenheiro Agrônomo José Paulo Guadagnin, e aos demais pesquisadores que auxiliaram na realização dos experimentos, pela cessão dos dados.

\section{Referências}

CARGNELUTTI FILHO, A.; STORCK, L. População de plantas na comparação do rendimento de grãos entre cultivares de milho. Pesquisa Agropecuária Brasileira, v.39, p.17-25, 2004.

CARGNELUTTI FILHO, A.; STORCK, L.; LUCIO, A.D. Ajustes de quadrado médio do erro em ensaios de competição de cultivares de milho pelo método de Papadakis. Pesquisa Agropecuária Brasileira, v.38, p.467-473, 2003a.

CARGNELUTTI FILHO, A.; STORCK, L.; LUCIO, A.D.; CARVALHO, M.P.; SANTOS, P.M. A precisão experimental relacionada ao uso de bordaduras nas extremidades das fileiras em ensaios de milho. Ciência Rural, v.33, p.607-614, 2003 b.

CARNEIRO, P.C.S. Novas metodologias de análise da adaptabilidade e estabilidade de comportamento. 1998. 168p. Tese (Doutorado) - Universidade Federal de Viçosa, Viçosa.

CONAGIN, A.; IGUE, T.; NAGAI, V. Tabelas para determinação do número de repetições no planejamento de experimentos. Campinas: Instituto Agronômico de Campinas, 1995. 17p. (Boletim Científico, 34).

CRUZ, C.D. Programa GENES - versão Windows: aplicativo computacional em genética e estatística. Viçosa: UFV, 2001. 648p.

CRUZ, C.D.; CARNEIRO, P.C.S. Modelos biométricos aplicados ao melhoramento genético. Viçosa: UFV, 2003. v.2.

CRUZ, V.F. da. Estudo sobre a correção de produção de parcelas em ensaios com milho. 1971. 143p. Tese (Doutorado) - Escola Superior de Agricultura Luiz de Queiroz, Piracicaba.

FEIJÓ, S.; STORCK, L.; LÚCIO, A.D.; LOVATO, C.; MARTIN, T.N. Melhoria da qualidade de ensaios de avaliação de cultivares de milho através de indicadores de fertilidade do solo. Ciência Rural, v.31, p.225-230, 2001.

LIN, C.S.; BINNS, M.R. A superiority measure of cultivar performance for cultivar x location data. Canadian Journal of Plant Science, v.68, p.193-198, 1988.

LOPES, S.J. Avaliação do efeito de diferentes formas de adubação sobre a precisão de ensaios de milho. 1993. 72p. Dissertação (Mestrado) - Universidade Federal de Santa Maria, Santa Maria.

LÚCIO, A.D. Parâmetros da precisão experimental das principais culturas anuais do Estado do Rio Grande do Sul. 1997. 64p. Dissertação (Mestrado) - Universidade Federal de Santa Maria, Santa Maria.
MARQUES, D.G. As pressuposições e a precisão dos ensaios de competição de cultivares de milho no Estado do Rio Grande do Sul. 1999. 42p. Dissertação (Mestrado) - Universidade Federal de Santa Maria, Santa Maria.

MORAIS, A.R.; OLIVEIRA, A.C.; CRUZ, J.C. Comparação de métodos de correção de produções de milho em parcelas experimentais. Relatório Técnico Anual do CNPMS: 1980-1984, p.130-132, 1986.

PIMENTEL-GOMES, F. A importância do número de repetições nos experimentos. Revista de Agricultura, v.69, p.243-245, 1994.

PIMENTEL-GOMES, F. Curso de estatística experimental. 13.ed. Piracicaba: Livraria Nobel, 1990. 467p.

RAMALHO, M.A.P.; FERREIRA, D.F.; OLIVEIRA, A.C. Experimentação em genética e melhoramento de plantas. Lavras: Ufla, 2000. 326p.

SCAPIM, C.A.; CARVALHO, C.G.P. de; CRUZ, C.D. Uma proposta de classificação dos coeficientes de variação para a cultura do milho. Pesquisa Agropecuária Brasileira, v.30, p.683-686, 1995.

SCHMILDT, E.R.; CRUZ, C.D.; ZANUNCIO, J.C.; PEREIRA, P.R.G.; FERRÃO, R.G. Avaliação de métodos de correção de estande para estimar a produtividade em milho. Pesquisa Agropecuária Brasileira, v.36, p.1011-1018, 2001.

STEEL, R.G.D.; TORRIE, J.H.; DICKEY, D.A. Principles and procedures of statistics. $3^{\text {rd }}$ ed. New York: McGraw Hill Book, 1997. 666p.

STORCK, L.; GARCIA, D.C.; LOPES, S.J.; ESTEFANEL, V. Experimentação vegetal. Santa Maria: UFSM, 2000a. 198p.

STORCK, L.; LOPES, S.J.; MARQUES, D.G.; TISOTT, C.A.; ROS, C.A. Análise de covariância para melhoria da capacidade de discriminação em ensaios de cultivares de milho. Pesquisa Agropecuária Brasileira, v.35, p.1311-1316, 2000b.

STORCK, L.; LÚCIO, A.D.; MARTIN, T.N.; LORENZ, L.H.; SOUZA, M.F. de. Diferenças de produtividade de grãos entre cultivares indicados de milho, ajustado para um mesmo número de plantas ou de espigas. Ciência Rural, v.32, p.745-750, 2002.

VENCOVSKY, R.; CRUZ, C.D. Comparação de métodos de correção do rendimento de parcelas com estandes variados - I: Dados simulados. Pesquisa Agropecuária Brasileira, v.26, p.647-657, 1991.

VERONESI, J.A.; CRUZ, C.D.; CORRÊA, L.A.; SCAPIM, C.A. Comparação de métodos de ajuste do rendimento de parcelas com estandes variados. Pesquisa Agropecuária Brasileira, v.30, p.169174, 1995.

ZUBER, M.S. Relative efficiency of incomplete block designs using corn uniform trial data. Journal of the American Society of Agronomy, v.34, p.30-47, 1942.

Recebido em 16 de abril de 2004 e aprovado em 22 de fevereiro de 2005 